\title{
Critical behavior of three-dimensional magnets with complicated ordering from three-loop renormalization-group expansions
}

\author{
A. I. Sokolov and K. B. Varnashev \\ Department of Physical Electronics, \\ Saint Petersburg Electrotechnical University, \\ Professor Popov Street 5, St.Petersburg, 197376, Russia
}

\begin{abstract}
The critical behavior of a model describing phase transitions in 3D antiferromagnets with $2 \mathrm{~N}$-component real order parameters is studied within the renormalizationgroup (RG) approach. The RG functions are calculated in the three-loop order and resummed by the generalized Padé-Borel procedure preserving the specific symmetry properties of the model. Anisotropic stable fixed point is found to exist in the RG flow diagram for $N \geq 2$ and lie near the Bose fixed point; corresponding critical exponents are close to those of the $X Y$-model. The accuracy of the results obtained is discussed and estimated.
\end{abstract}

64.60.Ak, 64.60.Fr, 75.40.Cx, 75.50.Ee

Typeset Using REVTEX 
In this Brief Report, we study within the field-theoretical RG approach in three dimensions (3D) the critical behavior of a model with three quartic coupling constants describing certain antiferromagnetic and structural phase transitions. The Hamiltonian of the model reads

$$
H=\int d^{3} x\left[\frac{1}{2}\left(m_{0}^{2} \varphi_{\alpha} \varphi_{\alpha}+\nabla \varphi_{\alpha} \nabla \varphi_{\alpha}\right)+\frac{u_{0}}{4 !}\left(\varphi_{\alpha} \varphi_{\alpha}\right)^{2}+\frac{v_{0}}{4 !} \varphi_{\alpha}^{4}+\frac{2 z_{0}}{4 !} \varphi_{2 \beta-1}^{2} \varphi_{2 \beta}^{2}\right],
$$

where $\varphi_{\alpha}$ is a real vector order parameter field, $\alpha=1,2, \ldots, 2 N, \beta=1,2, \ldots, N$. For $u_{0}=0$ Hamiltonian (0.1) describes $N$ non-interacting anisotropic $X Y$-models, for $z_{0}=0$ it reduces to that of the cubic model. When $N=2$ the expression (0.1) is relevant to the structural phase transition in the $\mathrm{NbO}_{2}$ crystal and, for $v_{0}=z_{0}$, to antiferromagnetic transitions in $T b A u_{2}$ and $D y C_{2}$. Another physically interesting case $N=3$ corresponds to antiferromagnetic phase transition in the $K_{2} \operatorname{IrCl}_{6}$ crystal and, for $v_{0}=z_{0}$, to those in $T b D_{2}$ and $N d$ 回].

This model was studied earlier by the $\epsilon$-expansion technique and directly in 3D within the lower (one- and two-loop) perturbative orders [1.2]. The main result of those investigations was the existence of the nontrivial (anisotropic) stable fixed point for $N \geq 2$ which gives rise to a new universality class with a certain set of critical exponents. The lower-order approximations, however, are known to lead to crude quantitative and, sometimes, to contradictory qualitative results, especially for systems with complicated symmetry [3]. That is 
why recently the critical behavior of the model (0.1) has been analyzed in the third order in $\epsilon$ [4]. Investigation of the fixed points stability and the calculation of marginal dimensionality $N_{c}$ of the order parameter separating two different regimes of critical behavior confirmed that the model (0.1) possesses the anisotropic stable fixed point for physically interesting cases $N=2$ and $N=3$. However, the eigenvalue exponents for this point turned out to be twofold degenerate in the one-loop approximation [5]. Such a degeneracy decreases markedly the accuracy expected within a given approximation and makes the calculating the eigenvalue exponents in higher orders in $\epsilon$ very difficult, while resummation of shorter series fails to provide proper numerical estimates. It is reasonable therefore to study the model (0.1) by means of some alternative approach. Below, the critical behavior of this model will be investigated by the RG technique in 3D in the three-loop approximation, aiming to improve existing qualitative and numerical results.

The character of the critical behavior is determined by the RG equations for quartic coupling constants. Calculating the $\beta$-functions entering their right-hand sides and critical exponents $\gamma$ and $\eta$ as functions of the dimensionless coupling constants $u, v$, and $z$ in the three-loop approximation, we obtain: 


$$
\begin{aligned}
& \beta_{u}=u\left\{1-u-\frac{1}{N+4}(3 v+z)+\frac{1}{27(N+4)^{2}}\left[2(41 N+95) u^{2}+300 u v+100 u z\right.\right. \\
& \left.+69 v^{2}+23 z^{2}\right]-\frac{1}{8(N+4)^{3}}\left[\left(5.39577 N^{2}+109.88075 N+199.64042\right) u^{3}\right. \\
& +(39.88127 N+493.84155) u^{2} v+(13.29376 N+164.61385) u^{2} z \\
& +(3.73134 N+302.86778) u v^{2}+58.35119 u v z+(1.24378 N+81.50553) \\
& \left.\left.\times u z^{2}+65.93728 v^{3}+3.73134 v^{2} z+21.97909 v z^{2}+9.35389 z^{3}\right]\right\}, \\
& \beta_{v}=v-\frac{1}{N+4}\left(6 u v+\frac{9}{2} v^{2}+\frac{1}{2} z^{2}\right)+\frac{1}{27(N+4)^{2}}\left[2(23 N+185) u^{2} v+624 u v^{2}\right. \\
& \left.+46 u v z+54 u z^{2}+231 v^{3}+23 v z^{2}+18 z^{3}\right]-\frac{1}{8(N+4)^{3}}\left[\left(-5.00443 N^{2}\right.\right. \\
& +83.70780 N+469.33398) u^{3} v+(4.47812 N+1228.60593) u^{2} v^{2} \\
& +(-7.50664 N+115.06965) u^{2} v z+(2.99978 N+98.15522) u^{2} z^{2} \\
& +957.78168 u v^{3}+4.47812 u v^{2} z+135.72564 u v z^{2}+60.68074 u z^{3} \\
& \left.+255.92974 v^{4}+4.10473 v^{2} z^{2}+49.83176 v z^{3}+5.05072 z^{4}\right], \\
& \beta_{z}=z\left\{1-\frac{1}{N+4}(6 u+3 v+2 z)+\frac{1}{27(N+4)^{2}}\left[2(23 N+185) u^{2}+462 u v\right.\right. \\
& \left.+262 u z+69 v^{2}+162 v z+41 z^{2}\right]-\frac{1}{8(N+4)^{3}}\left[\left(-5.00443 N^{2}+83.70780 N\right.\right. \\
& +469.33398) u^{3}+(-4.52123 N+934.14027) u^{2} v+(4.49249 N \\
& +507.69053) u^{2} z+411.65505 u v^{2}+550.60474 u v z+196.40638 u z^{2} \\
& \left.\left.+65.93728 v^{3}+108.78727 v^{2} z+118.33643 v z^{2}+21.85596 z^{3}\right]\right\} \\
& \gamma^{-1}=1-\frac{1}{4(N+4)}[2(N+1) u+3 v+z]+\frac{1}{4(N+4)^{2}}\left[2(N+1) u^{2}+6 u v+2 u z+3 v^{2}+z^{2}\right] \\
& -\frac{0.04813}{(N+4)^{3}}\left[4(N+1)^{2} u^{3}+18(N+1) u^{2} v+6(N+1) u^{2} z+6(N+4) u v^{2}+12 u v z\right. \\
& \left.+2(N+2) u z^{2}+9 v^{3}+3 v^{2} z+3 v z^{2}+z^{3}\right]-\frac{0.06182}{(N+4)^{3}}\left[4(N+1)(N+4) u^{3}\right. \\
& \left.+18(N+4) u^{2} v+6(N+4) u^{2} z+81 u v^{2}+18 u v z+21 u z^{2}+27 v^{3}+9 v z^{2}+4 z^{3}\right], \\
& \eta=\frac{2}{27(N+4)^{2}}\left[2(N+1) u^{2}+6 u v+2 u z+3 v^{2}+z^{2}\right]+\frac{0.00309}{(N+4)^{3}}\left[4(N+1)(N+4) u^{3}\right. \\
& \left.+18(N+4) u^{2} v+6(N+4) u^{2} z+81 u v^{2}+18 u v z+21 u z^{2}+27 v^{3}+9 v z^{2}+4 z^{3}\right] .
\end{aligned}
$$


To extract the physical information from these divergent series, the Padé-Borel resummation procedure will be used. Since the expansions of quantities depending on three variables $u, v$, and $z$ are dealt with, the Borel transformation is taken in a generalized form:

$$
f(u, v, z)=\sum_{i j k} c_{i j k} u^{i} v^{j} z^{k}=\int_{0}^{\infty} e^{-t} F(u t, v t, z t) d t, \quad F(x, y, w)=\sum_{i j k} \frac{c_{i j k} x^{i} y^{j} w^{k}}{(i+j+k) !} .
$$

To perform an analytical continuation, we address to the resolvent series

$$
\tilde{F}(x, y, w, \lambda)=\sum_{n=0}^{\infty} \lambda^{n} \sum_{l=0}^{n} \sum_{m=0}^{n-l} \frac{c_{l, m, n-l-m} x^{l} y^{m} w^{n-l-m}}{n !}
$$

which is a series in powers of $\lambda$ with coefficients being uniform polynomials in $u, v, z$, and then use Padé approximants $[L / M]$ in $\lambda$ at $\lambda=1$. The approximant [3/1] is employed for resummation of three-loop RG series Eqs. (0.2). The coordinates of all fixed points are determined for two most interesting cases $N=2$ and $N=3$. The results obtained are presented in Table I, which contains also, for comparison, analogous estimates found earlier from the two-loop RG series [2]. The global structure of the RG flows in the threeloop approximation is shown in Fig.1 (see the printed paper: Phys. Rev. B 59 (1999) 8363-8366).

The three-loop contributions to the $\beta$-functions are seen to affect the location of the fixed points considerably. Of special interest is the fortune of the nontrivial, anisotropic fixed point 8. In the one-loop approximation for $N=2$ it coincides with the Heisenberg fixed point 2 [6]. In higher orders this degeneracy is lifted out and the point 8 , remaining 
a stable one, moves toward the Bose fixed point 5 lying within the plane $v=z$. With increasing $N$, the former becomes closer and closer to the latter, indicating the tendency of the $O(2 N)$-symmetric interaction to vanish at criticality. This resembles the behavior of the cubic model that is known to split into $n$ non-interacting Ising models when $n \rightarrow \infty$.

The resummation procedure changes the perturbative expansions for $\beta$-functions by the complicated non-polynomial expressions. Does this procedure preserve the symmetry properties of the system? The model (0.1) possesses, apart from usual, the special symmetry properties which have been already used for testing a validity of approximations employed [2].

For example, if the field $\varphi_{\alpha}$ undergoes the transformation $\varphi_{2 \beta-1} \rightarrow \frac{1}{\sqrt{2}}\left(\varphi_{2 \beta-1}+\varphi_{2 \beta}\right), \varphi_{2 \beta} \rightarrow$ $\frac{1}{\sqrt{2}}\left(\varphi_{2 \beta-1}-\varphi_{2 \beta}\right)$, the coupling constants are also transformed:

$$
u \rightarrow u, \quad v \rightarrow \frac{1}{2}(v+z), \quad z \rightarrow \frac{1}{2}(3 v-z)
$$

but the structure of the Hamiltonian itself remains the same. Since such a transformation does not affect the RG equations, it can, at most, rearrange the fixed points leaving their coordinates unchanged [2,3, 迆. To check up whether such an invariance holds for the fixed points found, let us apply the transformation (0.7) to the content of Table I. Then points 1, 2, 5 and 8 will stay at their places while the coordinates of points 3 and 4 will turn into those of points 6 and 7 respectively and vice versa with accuracy of order of $10^{-4}$. Since the fixed point coordinates were evaluated numerically with just the same accuracy, it means 
that our resummation procedure exactly reproduces the symmetry properties discussed.

Let us determine further the critical exponents. The coefficients of the series for $\eta$ rapidly diminish, therefore its value may be found by direct substitution of the fixed point coordinates into the expansion (0.4). In the case of susceptibility exponent corresponding series (0.3) have to be resummed. Using the generalized Padé-Borel procedure, we evaluate the exponent $\gamma$ and then, addressing the values of $\eta$, calculate critical exponent $\alpha$ by the scaling relations. The results for $N=2$ and $N=3$ are presented in Table II. As is seen, the critical exponents of the fixed point 8 only slightly differ from those of the Bose one reflecting a closeness of both fixed points in the $(u, v, z)$ space.

The critical exponents for the fixed points 2 (Heisenberg), 5 (Bose), and 3 (Ising) thus found are close to their high-precision analogs resulting from the six-loop RG expansions [8]; the differences do not exceed 0.02-0.03. For the anisotropic fixed point 8 the situation is more complicated. In the three-loop approximation it is three-dimensionally stable and characterized by negative value of the specific heat exponent $\alpha$, while the Bose fixed point looks unstable since it has a positive $\alpha$. In fact, however, the $X Y$-like critical behavior in 3D is described by the negative $\alpha$ as is known both from the six-loop RG calculations [8] and recent extremely accurate measurements [9]. Moreover, a sign of the exponent $\alpha$ at the Bose fixed point determines either the $O(2 N)$-symmetric interaction is relevant near this point or not [10]: if $\alpha<0$, the Bose fixed point should be stable with respect to this interaction. It 
means that what is really stable is the fixed point 5 while the stability of the fixed point 8 within the plane $v=z$ is an artifact of the three-loop approximation.

Keeping this in mind we can estimate an accuracy of the anisotropic fixed point coordinates given by the three-loop RG series. Because of the obvious topological reasons (see Fig.1), the point 8 , being in fact unstable, should have $u_{c}<0$. At the same time, since the true value of $\alpha$ at the Bose fixed point is very small [8,9] the points 5 and 8 should be very close one to another and the modulus of $u_{c}$ for the point 8 should be very small as well. Hence, as can be deduced from the numbers in the last column of Table I, the three-loop approximation predicts locations of this point for $N=2$ and $N=3$ with errors about 0.2 and 0.1 respectively.

Dealing with the theory without a small parameter and the short (three-loop) perturbative series one would refer to such an accuracy as satisfactory. On the other hand, numerically small errors lead in this case to qualitatively incorrect results making the situation rather unfavorable. The point is that in $3 \mathrm{D}$ the model (0.1) is almost identical to some marginal system for which $\alpha=0$ at the $X Y$-like criticality and, as a consequence, fixed points 5 and 8 coincide. Since such a marginality is far from to be seen in the one-loop approximation, it hardly manifests itself within a perturbation theory, even in higher orders.

The "near-marginality" is not a unique feature of the model (0.1) being typical for 3D systems with several coupling constants. Thus, for the cubic model the marginal value of $n$ 
that separates two different regimes of critical behavior is numerically close to the physical value $n=3$ [11]. The model describing phase transitions into chiral states has the marginal dimensionality of the complex order parameter $N_{c 2}$ (separating domains of continuous and first-order transitions) that is also very close to the physical value $N=2$ [3], etc. Hence, to answer the question about the type of the critical behavior of such "unconvenient" models the higher-order RG analysis should be carried out.

The calculation of the RG functions of the model (0.1) in the next, four-loop approximation is an extremely difficult problem. The point discussed, however, may be cleared up without performing additional RG calculations. Indeed, let us trace how the numerical value of the exponent $\alpha$ for the Bose fixed point depends on the order of the RG approximation. The estimates for $\alpha$ obtained in the one-, two-, three-, four-, five-, and six-loop orders using the Padé-Borel resummation technique are $0.125,-0.012,0.009,-0.007,-0.007$, and -0.007 , respectively. As is seen, the sign of exponent $\alpha$ alternates up to four-loop order and then stays negative indicating the stability of the Bose fixed point. Since two neighbouring fixed points can not be stable simultaneously we conclude that the fixed point 8 should be unstable within the four-loop and higher-order approximations. Consequently, the next, four-loop RG approximation will be sufficient to yield the correct structure of the RG flow diagram as well as high-precision numerical estimates for the critical exponents. 


\section{REFERENCES}

[1] D. Mukamel, Phys. Rev. Lett. 34, 481 (1975); D. Mukamel and S. Krinsky, J. Phys. C 8, L496 (1975) and Phys. Rev. B 13, 5065, 5078 (1976).

[2] K. B. Varnashev, A. I. Sokolov, Fiz. Tverd. Tela 38, 3665 (1996) [Phys. Solid State 38, 1996 (1996)].

[3] S. A. Antonenko and A. I. Sokolov, Phys. Rev. B 49, 15901 (1994); S. A. Antonenko, A. I. Sokolov, K. B. Varnashev, Phys. Lett. A 208, 161 (1995).

[4] A. I. Mudrov and K. B. Varnashev, Phys. Rev. B 57, 3562 (1998).

[5] A. I. Mudrov and K. B. Varnashev, Phys. Rev. B 57, 5704 (1998).

[6] In the one-loop approximation for $N=2$ the Heisenberg fixed point is fourfold degenerate [2]. So strong degeneracy, being typical for a number of complicated models [3,,7], is in fact occasional and lifted out in the higher-order approximations.

[7] S. A. Brazovskii, I. E. Dzyaloshinskii, B. G. Kukharenko, Zh. Eksp. Teor. Fiz. 70, 2257 (1976) [Sov. Phys. JETP 43, 1178 (1976)]; J.-C. Toledano, L. Michel, P. Toledano, and E. Brezin, Phys. Rev. B 31, 7171 (1985).

[8] G. A. Baker, B. G. Nickel, and D. I. Meiron, Phys. Rev. B 17, 1365 (1978); J. C. Le Guillou and J. Zinn-Justin, Phys. Rev. B 21, 3976 (1980); S. A. Antonenko and A. I. 
Sokolov, Phys. Rev. E 51, 1894 (1995).

[9] S. Goldner and G. Ahlers, Phys. Rev. B 45, 13129 (1992); J. A. Lipa, D. R. Swanson, J. A. Nissen, T. C. P. Chui, and U. E. Israelsson, Phys. Rev. Lett. 76, 944 (1996).

[10] J. Sak, Phys. Rev. B. 10, 3957 (1974); R. A. Cowley and A. D. Bruce, J. Phys. C. 11, 3577 (1978).

[11] I. O. Mayer, A. I. Sokolov, and B. N. Shalayev, Ferroelectrics 95, 93 (1989); H. Kleinert, V. Schulte-Frohlinde, Phys. Lett. B 342, 284 (1995); B. N. Shalaev, S. A. Antonenko, A. I. Sokolov, Phys. Lett. A 230, 105 (1997). 


\section{FIGURES}

FIG. 1. Three-dimensional flow diagram of the RG equations for $N=2$ in the three-loop approximation. 


\section{TABLES}

TABLE I. Coordinates of the fixed points of the RG equations for $N=2$ and $N=3$ obtained within three-loop ([3/1]) and two-loop ([2/1]) approximations.

\begin{tabular}{|c|c|c|c|c|c|c|c|c|c|}
\hline & & 1 & 2 & 3 & 4 & 5 & 6 & 7 & 8 \\
\hline \multicolumn{10}{|c|}{$\mathrm{N}=2$} \\
\hline \multirow[t]{2}{*}{$u_{c}$} & {$[3 / 1]$} & 0.0 & 1.3671 & 0.0 & 0.9254 & 0.0 & 0.0 & 0.9255 & 0.1872 \\
\hline & {$[2 / 1]^{*}$} & 0.0 & 1.4863 & 0.0 & 1.0166 & 0.0 & 0.0 & 1.0166 & 0.0332 \\
\hline \multirow[t]{2}{*}{$v_{c}$} & {$[3 / 1]$} & 0.0 & 0.0 & 1.8883 & 0.7764 & 1.6833 & 0.9442 & 0.3882 & 1.4914 \\
\hline & {$[2 / 1]^{*}$} & 0.0 & 0.0 & 2.1289 & 0.8358 & 1.8700 & 1.0645 & 0.4178 & 1.8344 \\
\hline \multirow[t]{2}{*}{$z_{c}$} & {$[3 / 1]$} & 0.0 & 0.0 & 0.0 & 0.0 & 1.6833 & 2.8325 & 1.1646 & 1.4914 \\
\hline & {$[2 / 1]^{*}$} & 0.0 & 0.0 & 0.0 & 0.0 & 1.8700 & 3.1934 & 1.2537 & 1.8344 \\
\hline \multicolumn{10}{|c|}{$\mathrm{N}=3$} \\
\hline \multirow[t]{2}{*}{$u_{c}$} & {$[3 / 1]$} & 0.0 & 1.3310 & 0.0 & 0.6005 & 0.0 & 0.0 & 0.6005 & 0.0780 \\
\hline & {$[2 / 1]^{*}$} & 0.0 & 1.4262 & 0.0 & 0.6307 & 0.0 & 0.0 & 0.6307 & 0.0094 \\
\hline \multirow[t]{2}{*}{$v_{c}$} & {$[3 / 1]$} & 0.0 & 0.0 & 2.2030 & 1.4971 & 1.9639 & 1.1015 & 0.7485 & 1.8845 \\
\hline & {$[2 / 1]^{*}$} & 0.0 & 0.0 & 2.4837 & 1.6855 & 2.1816 & 1.2419 & 0.8428 & 2.1716 \\
\hline \multirow[t]{2}{*}{$z_{c}$} & {$[3 / 1]$} & 0.0 & 0.0 & 0.0 & 0.0 & 1.9639 & 3.3045 & 2.2456 & 1.8845 \\
\hline & {$[2 / 1]^{*}$} & 0.0 & 0.0 & 0.0 & 0.0 & 2.1816 & 3.7256 & 2.5283 & 2.1716 \\
\hline
\end{tabular}

* Quoted from Ref. [2] 
TABLE II. Critical exponents for $N=2$ (upper lines) and $N=3$ (lower lines) calculated within three-loop approximation.

\begin{tabular}{|c|c|c|c|c|c|c|c|c|}
\hline & 1 & 2 & 3 & 4 & 5 & 6 & 7 & 8 \\
\hline \multirow[t]{2}{*}{$\gamma$} & 1 & 1.4260 & 1.2406 & 1.3989 & 1.3098 & 1.2406 & 1.3990 & 1.3360 \\
\hline & 1 & 1.5164 & 1.2406 & 1.4080 & 1.3098 & 1.2406 & 1.4080 & 1.3291 \\
\hline \multirow[t]{2}{*}{$\eta$} & 0.0 & 0.0257 & 0.0246 & 0.0258 & 0.0260 & 0.0246 & 0.0258 & 0.0261 \\
\hline & 0.0 & 0.0238 & 0.0246 & 0.0253 & 0.0260 & 0.0246 & 0.0253 & 0.0261 \\
\hline \multirow[t]{2}{*}{$\alpha$} & 0.0 & -0.1669 & 0.1159 & -0.1258 & 0.0094 & 0.1159 & -0.1259 & -0.0305 \\
\hline & 0.0 & -0.3020 & 0.1159 & -0.1390 & 0.0094 & 0.1159 & -0.1390 & -0.0199 \\
\hline
\end{tabular}

